\title{
Localization of the Sound Source with the Use of the First-order Ambisconic Microphone
}

\author{
J. WierzBicki, P. MaŁecki AND J. WiciaK* \\ AGH - University of Science and Technology, Department of Mechanics and Vibroacoustics \\ Department of Mechanics and Vibroacoustics, al. A. Mickiewicza 30, 30-059 Krakow, Poland
}

\begin{abstract}
The paper presents results of determination of sound wave direction based on signals from first-order ambisonic microphone. The experiment consisted in recording the test signals with the SoundField microphone positioned in the axis of the turntable in the anechoic chamber. The spherical coordinates of the sound intensity vector were calculated with use of three different conceptual and numerical approaches and were compared to actual values resulting from the geometry of the system. Accuracy of the localization of the sound source depending on the frequencies and the method for determination of the spherical coordinates as well as on the time constant and the parameters of the signal recording was presented. The obtained results show the effectiveness of the calculation methods used for localization of the sound source.
\end{abstract}

DOI: 10.12693/APhysPolA.123.1114

PACS: 43.58.Vb, 43.60.Jn, 43.20.Ye

\section{Introduction}

There are several known methods for sound source localization. There are methods based on time difference and level deference. The article focuses on energy-based localization method which employs measurement of the sound intensity vector. Sound intensity can be measured using intensity probes [1] but it needs a lot of effort and is very expensive. The first-order ambisonic microphone allows to estimate the direction of sound source and by using two of them it is possible to estimate sound source exact position. Accuracy of triangulation depends on precision of angle estimation. The main aim of the research is to estimate the maximal accuracy of localization of the sound source with the use of the ambisonic microphone in laboratory conditions.

\section{The aim and course of the test}

The measurement consisted in recording the test signals with SoundField (SF) microphone positioned in the axis of the turntable in the big anechoic chamber at the AGH-UST. The SF ST350 transducer by English company SoundField Ltd. is a micro-matrix of microphones (capsules) with cardioidal directional characteristics positioned in geometrical centers of triangle walls of the regular tetrahedron. Due to location of the capsules and the method for processing the signals that allows treatment of the microphones as coincidental, it is called the first-order ambisonic microphone. After application of relevant algorithms, out of signals from four capsules the signals are obtained that correspond to the microphones

*corresponding author; e-mail: wierzbic@agh.edu.pl with bi-directional characteristics positioned along the $X, Y$, and $Z$ axes, denoted so by analogy to the directions, and one signal that corresponds to the microphone with omnidirectional characteristics denoted $W$. The typical application of such kind of microphone is recording and transmission of the spatial sound for the radio and television production. It can also be used during measurements of the spatial impulse response [2].

The test signals were emitted continuously by the active speaker Genelec 8030 located at the distance of $4.5 \mathrm{~m}$ from the rotation axis of the microphone. The schematic of the measurement stand is presented in Fig. 1.

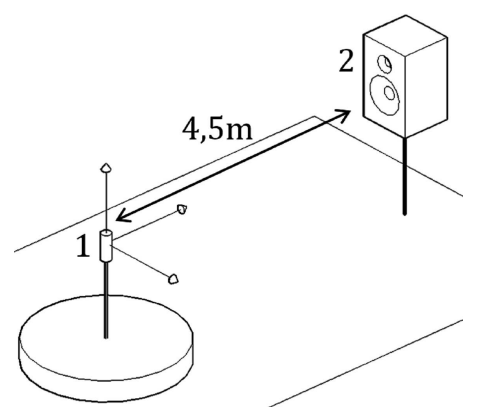

Fig. 1. The schematic of the measurement stand, where 1 denotes SF microphone and 2 sound source.

The measurement was performed with very small rotational speed of the turntable that was $0.0093 \mathrm{rad} / \mathrm{s}=$ $0.089 \mathrm{rpm}$. The rotation time was $11 \mathrm{~min}$ and $14 \mathrm{~s}$ per one revolution. The signal was recorded with sampling frequency $96 \mathrm{kHz}$ at 24-bit quantization with the use of RME Fireface 800 converter. The test signals consisted of the tones with frequencies from $16 \mathrm{~Hz}$ to $16 \mathrm{kHz}$ spaced by one octave and the white noise. Based on the recorded signals angular position of the microphones related to the source was calculated and compared to the actual posi- 
tion. The calculation was performed with use of different time constants and parameters of the recorded signal. Also the alternative methods for calculation of the searched values were used.

The microphone was installed on the typical stand at height of $1.2 \mathrm{~m}$ and in the "InVision" microphone grip (www.rycote.com/products/invision studio_kit/invision_studio/), with special construction that did not introduce significant distortions into the acoustic field.

The calculation was performed by estimation of the sound intensity vector $\boldsymbol{I}$ that is defined by the equation (1). Having the B-format signal components $(W, X, Y, Z)$, one can calculate its approximate value [3]:

$$
I=p u,
$$

where the acoustic pressure value $p$ and the velocity vector $\boldsymbol{u}$ can be estimated as follows:

$$
\begin{aligned}
& p=\sqrt{2} W \\
& \boldsymbol{u}=\left[\begin{array}{l}
\mathrm{X} \\
\mathrm{Y} \\
\mathrm{Z}
\end{array}\right] .
\end{aligned}
$$

Based on the value of the intensity vector $I=\left[I_{x}, I_{y}, I_{z}\right]$, one can calculate the spherical coordinates $\varphi$ and $\psi$, which in this case correspond to the position of the sound source:

$$
\begin{aligned}
& \varphi=\arg \left(I_{x}+\mathrm{i} I_{y}\right), \\
& \psi=\arg \left(\sqrt{I_{x}^{2}+I_{y}^{2}}+\mathrm{i} I_{z}\right) .
\end{aligned}
$$

The values of the signal $[W, X, Y, Z]$ should be calculated with assumption of the steady state in the analyzed timeframe. The value of the signal in given time frame can be calculated with use of different conceptual and numerical approaches:

1) Calculation of the RMS of particular components for timeframes and phase analysis for determination of the direction of the spherical coordinates [4]:

$$
\left\|I_{x}\right\|=\sqrt{\sum_{n} \frac{1}{N} x^{2}(n)},
$$

2) Determination of the sum from the relevant products of particular signal samples in the given time frame [5]:

$$
\left\|I_{x}\right\|=\sum_{n} x(n) \cdot w(n),
$$

3) Calculation of the sum of the consecutive ambisonic components taking into account the phase of the $W$ component:

$$
\left\|I_{x}\right\|=x(n) \cdot \operatorname{sign}(w(n)) .
$$

Using the specified algorithms, the spherical coordinates of the vector of sound intensity were calculated for consecutive tested signals and compared to actual values resulting from geometry of the system.

\section{Preliminary analysis of the results}

Figure 2 shows the values of the rotation angle in time, calculated based on the sample recorded signal. Only horizontal angle is shown because axis of rotation is perpendicular to $\mathrm{SF} Z$ component so the vertical angle should be constant. The lines in the chart represent the different methods for calculation of the searched angle (presented in the previous section). Although the relationship in no case is linear, it is characterized by noticeable regularity. Figure 3, as a zoomed part of Fig. 2, shows more details and differences between the calculated curves. Figure 2 does show any difference between methods 2 and 3 . This results from the low resolution of differences between the results.

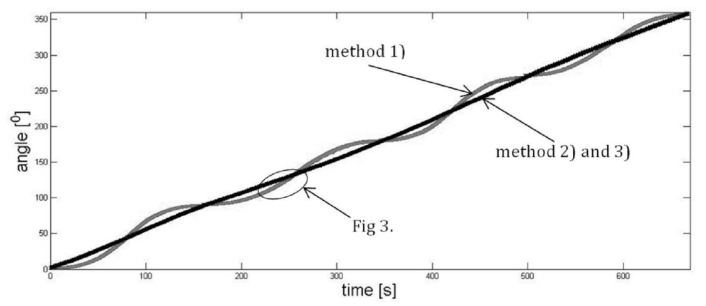

Fig. 2. The calculated values of the horizontal angle with use of different methods for $1 \mathrm{kHz}$ tone and one full revolution of the microphone around its axis.

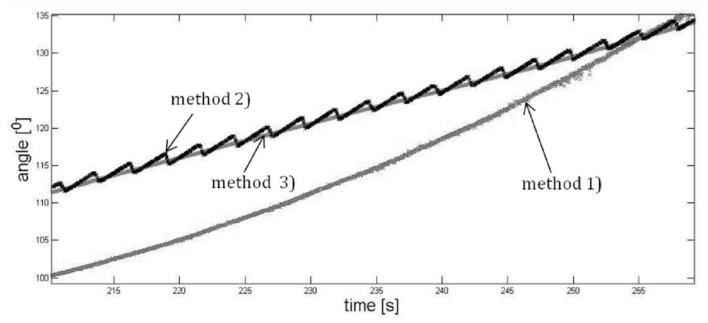

Fig. 3. Values of the horizontal angle calculated with the use of different methods for $1 \mathrm{kHz}$ tone and the movement of microphone in the angle of $35^{\circ}$.

From Figs. 2 and 3 it can be concluded that the calculated points, representing the angular position of the microphone, have a relatively small local variation related to different global regular curves. Such global nonlinearities might be approximated by the polynomial fitting of the obtained data. After approximation with the mean square method, by iterative increase of the rank of the approximation it was determined that the variation of the matching error for the polynomial in the degree of 9. For the consecutive signals, comparison was made between the measured values and the actual angle of the microphone on the turntable between the measured values and the curve defined by the polynomial determined in the approximation procedure. In order to compare this two variants, the standard deviation was calculated 
(for both the actual value and the polynomial approximation). The distribution of the measured values with respect to actual values and approximated values estimates the accuracy with which the position of the source is measured, both for actual values and for polynomial, which potentially could compensate the noticed non-linearities. Figure 4 presents sample result of the approximation.

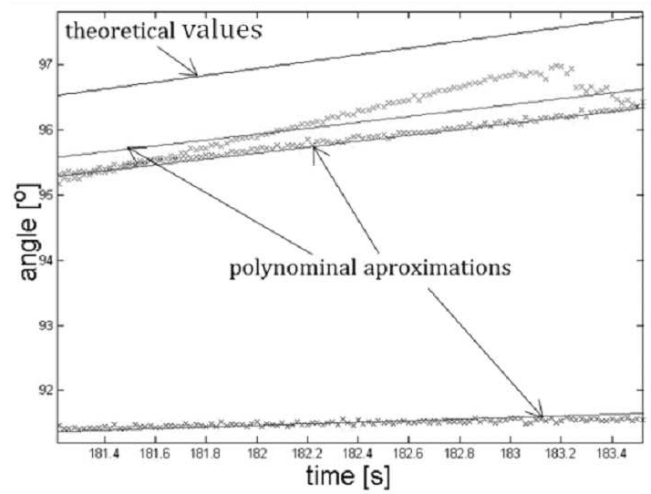

Fig. 4. Graphical presentation of the curves determined in approximation of the measured values and actual values of the measured horizontal angle.

\section{Accuracy of localization of the sound source depending on frequencies and the method used for determination of the component signals}

Table I presents the standard deviation of the measured values with respect to actual values and to the polynomial approximation. As the test signals, the tones of different frequencies and the white noise were used. The measurement was performed for the averaging time of $0.02 \mathrm{~s}$.

TABLE I

Standard deviation $\sigma\left[{ }^{\circ}\right]$ of the measured values from actual values (denoted ${ }^{*}$ ) and approximation of the results with polynomial (denoted ${ }^{* *}$ ) for 3 methods of calculation of localization of the sound source presented in Sect. 1 for different frequency $f$ of test signals. WN denotes white noise.

\begin{tabular}{c|r|r|r|r|r|r|r|r|r|r}
\hline \hline $\begin{array}{c}\sigma \text { and } \\
\text { approach }\end{array}$ & \multicolumn{8}{c}{$\sigma$ for different numerical approaches } \\
and signal frequencies \\
\cline { 2 - 10 }$\sigma 1^{*}$ & 39.2 & 10.9 & 10.7 & 10.6 & 9.3 & 9.2 & 10.6 & 43.2 & 92.5 & 41.7 \\
$\sigma 1^{* *}$ & 24.7 & 2.7 & 1.3 & 1.2 & 1.2 & 1.0 & 1.7 & 26.0 & 59.3 & 19.6 \\
$\sigma 2^{*}$ & 23.5 & 4.7 & 4.1 & 3.7 & 2.8 & 3.5 & 3.8 & 32.5 & 96.5 & 10.7 \\
$\sigma 2^{* *}$ & 20.1 & 0.8 & 0.2 & 0.1 & 0.1 & 0.2 & 0.7 & 15.1 & 87.5 & 8.9 \\
$\sigma 3^{*}$ & 26.4 & 4.7 & 4.1 & 3.7 & 2.8 & 3.5 & 3.8 & 53.7 & 87.5 & 16.2 \\
$\sigma 3^{* *}$ & 21.1 & 0.8 & 0.2 & 0.1 & 0.2 & 0.5 & 0.9 & 40.7 & 87.1 & 13.5
\end{tabular}

Based on Table I one can conclude that localization of the sound source with use of SF microphone in not effective for high frequencies. For frequencies above $4 \mathrm{kHz}$, the standard deviation is very high for each of the calculation method, both for the approximation and for the theoretical values. The results obtained by methods 2 and 3 are similar and, except for $125 \mathrm{~Hz}$, smaller deviation is achieved for method 2. Method 1 generated the largest standard deviation both in terms of average value and the approximation with polynomial.

\section{Accuracy of localization of the sound source depending on the time constant and parameters of the signal recording}

The effect of the values of the amplitudes in particular directions in time, was examined for the frequency of $500 \mathrm{~Hz}$ for which one of the best results was obtained in the list in Table I. There were 5 different time constants used, and the results are gathered in Table II.

TABLE II

The effect of the time constant on the accuracy of localization of the sound source with the use of the first-order ambisonic microphone. Standard deviation of the measured values from actual values $\left(\right.$ denoted $\left.^{*}\right)$ and approximation of the results with polynomial (denoted ${ }^{* *}$ ).

\begin{tabular}{c|c|c|c|c|c}
\hline \hline$\sigma$ & \multicolumn{5}{|c}{ Signal timeframe [s] } \\
\cline { 2 - 6 } & 0.002 & 0.02 & 0.125 & 0.25 & 1 \\
\hline$\sigma 2^{*}$ & 4.0 & 3.7 & 3.7 & 3.8 & 2.9 \\
$\sigma 2^{* *}$ & 1.6 & 0.1 & 0.0 & 0.0 & 0.0
\end{tabular}

TABLE III

The effect of the parameters of the signal on the accuracy of localization of the sound source with the first-order ambisonic microphone. Standard deviation of the measured values from actual values ((denoted $\left.{ }^{*}\right)$ and approximation of the results with polynomial (denoted $\left.{ }^{* *}\right)$.

\begin{tabular}{c|c|c|c|c}
\hline \hline$\sigma$ & \multicolumn{4}{|c}{ Recording parameters } \\
\hline & $96 \mathrm{k} \mathrm{24}$ bit & $96 \mathrm{k} 16$ bit & $48 \mathrm{k} 24$ bit & $22.05 \mathrm{k} 24 \mathrm{bit}$ \\
\hline$\sigma 2^{*}$ & 3.8 & 3.8 & 3.7 & 3.7 \\
$\sigma 2^{* *}$ & 0.1 & 0.1 & 0.1 & 0.1
\end{tabular}

Recording of the signals was performed with the sampling frequency $96 \mathrm{kHz}$ and 24-bit quantization. able III shows the effect of resampling and requantization of the signal on the analyzed angular accuracy for the frequency $500 \mathrm{~Hz}$ and the time constant $0.02 \mathrm{~s}$.

\section{The uncertainty of the results}

Attention should be focused on the geometrical factor which, in the opinion of authors, may significantly affect the uncertainty of the performed measurement. The lack of the proper positioning mechanism and/or possibility to measure precisely the deviation of the microphone from the axis of the turntable introduces the occurrence 
of an eccentricity which distorts the reading of the angle position as compared to the theoretical geometry of the system. Figure 5 presents trigonometric analysis of the effect of eccentricity on direction towards the fixed source from which it follows that

$$
\beta=\tan ^{-1}\left(\frac{d \sin \alpha}{d \cos \alpha+e}\right) \text {. }
$$

Based on Eq. (9) and Fig. 5, for the distance from the source $d=4.5 \mathrm{~m}$ and eccentricity $e=1 \mathrm{~cm}$, the calculated angle $\beta$ might differ from the actual value $\alpha$ by no more than one degree (from the theoretical value). One should note that the resulting error would have a regular character.

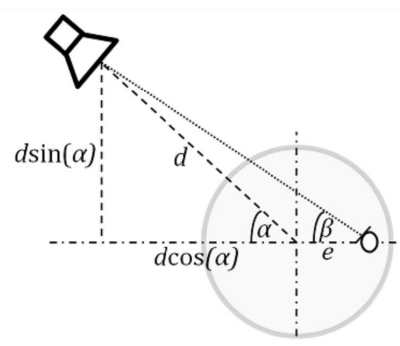

Fig. 5. Geometric analysis of the effect of the eccentricity on the accuracy of reading the horizontal angle.

\section{Summary and conclusion}

The performed research allows to plan for subsequent stages of work on the methods for localization of the sound sources with the use of the first-order ambisonic microphone. The performed research indicates high precision of the localization of the sound source in the laboratory conditions for mid-range frequencies. The obtained results show the effectiveness of the calculation methods used for localization of the sound source. The best method is to multiply the pressure component by the directional component. Using directional component values only gives almost as good results as the previous method but much better than calculating RMS values which is the very common approach in similar applications.

They allow also the use of the corrections for compensation of the errors of applied methods. The prepared experiments due to application of localization in open space concern problem of calibration and uncertainty evaluation for SoundField microphone with all-weather housing [6].

\section{References}

[1] S. Weyna, Arch. Acoust. 28, 191, (2003).

[2] P. Małecki, J. Wiciak, J. Wierzbicki, Acta Phys. Pol. A 121, A-110 (2012).

[3] J. Merimaa, V. Pulkki, J. Audio Eng. Soc. 53, 1115 (2005).

[4] C.A. Dimoulas, G.M. Kalliris, K.A. Avdelidis, G.V. Papanikolaou, Proc. 126th AES Convention, Audio Engineering Society, New York 2009, Paper 7691.

[5] F. Menzer, C. Faller, Proc. 125th AES Convention, New York 2008, Paper 7614.

[6] G. Wszolek, Acta Phys. Pol. A 119, 1081, (2011). 